\title{
Robust Repetitive Controller for Fast AFM Imaging
}

\author{
Serkan Necipoglu, Selman A. Cebeci, Yunus E. Has, Levent Guvenc, and Cagatay Basdogan
}

\begin{abstract}
Currently, atomic force microscopy (AFM) is the most preferred scanning probe microscopy method due to its numerous advantages. However, increasing the scanning speed and reducing the interaction forces between the probe's tip and the sample surface are still the two main challenges in AFM. To meet these challenges, we take advantage of the fact that the lateral movements performed during an AFM scan are a repetitive motion and propose a repetitive controller (RC) for the $z$-axis movements of the piezoscanner. The $\mathrm{RC}$ utilizes the profile of the previous scan line while scanning the current line to achieve a better scan performance. The results of the scanning experiments performed with our AFM setup show that the proposed RC significantly outperforms a conventional PI controller that is typically used for the same task. The scan error and the average tapping forces are reduced by $66 \%$ and $58 \%$, respectively, when the scanning speed is increased by sevenfold.
\end{abstract}

Index Terms-Atomic force microscopy (AFM), nanoscanning, repetitive controller ( $\mathrm{RC})$, system identification.

\section{INTRODUCTION}

$\mathbf{T}$ HE use of atomic force microscopy (AFM) in many different applications has increased rapidly since its invention in 1986 [1]. It has several advantages over the other scanning microscopy methods such as SEM and scanning tunneling microscopy. These include easy sample preparation, the ability to scan surfaces in air, liquid, and vacuum environments, and relatively lower cost. For this reason, the AFM has a wide range of use in material science, electronics, optics, semiconductor industry, biology, and other areas of life sciences. The primary components of an AFM setup operating in dynamic mode are illustrated in Fig. 1. The sample to be scanned is placed on a 3-DOF piezoactuated stage. In tapping mode AFM operation, first the scanning probe is excited to vibrate sinusoidally near the resonance frequency in free air and then brought close to the sample to lightly tap its surface. In amplitude modulation scheme, the tapping amplitude of the probe $A_{\text {act }}$ is kept at a set value $A_{\text {set }}$ by a feedback controller adjusting the vertical (z-axis) movements of the stage while the scan proceeds on the lateral axes $(x$ and $y$ ). This lateral motion is either triangular

Manuscript received July 21, 2010; revised December 8, 2010; accepted January 10, 2011. Date of publication January 17, 2011; date of current version September 8, 2011. The review of this paper was arranged by Associate Editor L. Dong.

S. Necipoglu is with the Department of Mechanical Engineering, Istanbul Technical University, Istanbul 34437, Turkey (e-mail: necipoglu@itu.edu.tr).

S. A. Cebeci, Y. E. Has, and C. Basdogan are with Robotics and Mechatronics Laboratory, College of Engineering, Koc University, Istanbul 34450, Turkey (e-mail: secebeci@ku.edu.tr, yhas@ku.edu.tr, cbasdogan@ku.edu.tr).

L. Guvenc is with the Department of Mechanical Engineering, Okan University, Istanbul, Turkey (e-mail: levent.guvenc@ okan.edu.tr).

Color versions of one or more of the figures in this paper are available online at http://ieeexplore.ieee.org.

Digital Object Identifier 10.1109/TNANO.2011.2106797

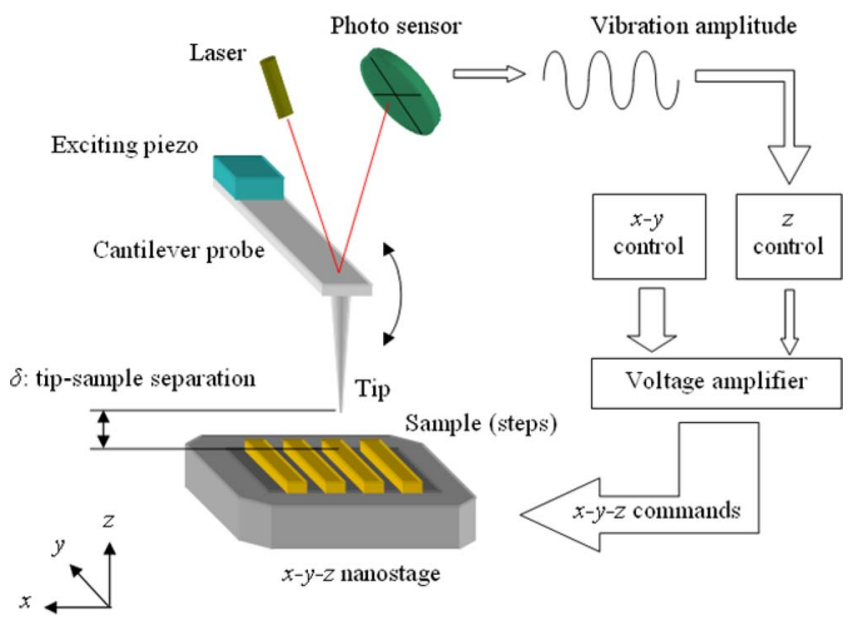

Fig. 1. Dynamic-mode AFM: the probe is excited by a piezoelectric element to vibrate sinusoidally near the resonance frequency and its vibration amplitude is measured by a laser beam reflected on a photosensor. The sample to be scanned is placed on a piezoactuated nanostage. Typically, the controller used for the lateral scan motion on the $x-y$ plane is open loop, while a closed-loop controller is used for the $z$ motion to acquire the surface topography based on the vibration amplitude of the probe.

or quadratic and controlled separately. Typically, PID-type controllers have been used for controlling the vertical and lateral movements of the stage [2]. The movement of the stage along the vertical axis (z-axis) is recorded as the surface height for each grid point on the $x-y$ plane to construct a topographic map of the scanned surface.

Increasing the scanning speed and reducing the magnitude of the interaction forces between the probe tip and the sample surface are two main challenges in AFM scanning. For example, it takes several minutes to scan even a small area of $2 \mu \mathrm{m} \times 2$ $\mu \mathrm{m}$. Besides, AFM is too slow to capture the phases of some rapid biological phenomena and new high-speed imaging techniques (see the review in [3]) have already shown to be highly effective [4], [5]. Moreover, during the scan process, the sample and the probe can be damaged easily if the magnitude of the interaction forces is high. This is especially critical when scanning biological samples since high interaction force can cause an irreversible damage on the sample.

One of the major limitations on the scanning speed of an AFM is the bandwidth of the piezoactuated stage. The physical bandwidth is limited by the resonance frequency of the mechanical structure, where the control bandwidth is limited by the gain margin of the closed-loop system commanding the stage. One way to obtain a higher scanning speed is to improve the mechanical design of the stage [6]. The other is to extend the control bandwidth by using a more sophisticated controller instead of the conventional PID controller. For example, feedback controllers based on $H_{\infty}$ theory are proposed in [7] and [8] 
for adjusting the movements of the stage in the $z$-axis and the lateral axes. A scanning speed that is five times higher than that of the conventional PID controller is reached in [7]. An inversion-based feedforward controller is presented in [9] for improving the tracking performance of the stage in lateral axes at high frequency. A detailed comparison of various feedback and/or feedforward controllers used for the lateral motion is given in [10]. In addition to adapting a more sophisticated controller, one can also take advantage of the fact that the successive scan lines are quite similar to each other to further improve the controller performance. For example, a PID feedback controller combined with a feedforward controller is presented in [11], where the profile of the previous scan line is supplied to the feedforward controller to improve the scanning speed. In [12], a more sophisticated $H_{\infty}$ feedback and feedforward controllers are used in tandem for the same purpose. In [13], a surface topography learning observer is designed by using a rule-based approach for perfect tracking control in vertical motion. The tracking error is improved by up to $80 \%$. An iterative learning controller (ILC) proposed in [14] improves the tracking performance of a contact mode AFM in vertical axis by eightfold at high scanning speeds. In [15], a repetitive controller (RC) is designed and implemented for controlling the lateral scan motion in AFM.

In this paper, we propose an RC for the dynamic-mode AFM, which can reject the repetitive disturbances (i.e., surface profile) successfully. This is due to the fact that the memory loop inside the $\mathrm{RC}$ becomes the generator of any repetitive input signal when the period of the memory loop is adjusted to match the period of the repetitive input signal [16]. Based on the internal model principle, the generator of the input signal on the forward path of a feedback loop drives the steady-state error to zero, and provides perfect disturbance rejection [17]. From another point of view, the RC introduces infinite loop gains at the fundamental frequency of the input signal and its harmonics [18], which is desirable, but may cause instabilities, for example, in some of the methods mentioned earlier. However, RC provides robust stability when it is used with appropriate filters [19]-[21].

The proposed $\mathrm{RC}$ is implemented for the tapping mode AFM and tested in our home-made AFM setup. Its performance is compared to that of the conventional PI controller. It is proposed as an add-on controller to a conventional PI controller. Hence, it can be turned on and off whenever necessary during the scan since its operation does not depend on the initial conditions as it does in ILC [22]. The main contributions of this study are itemized as follows.

1) In our implementation, we control the $z$-axis movements of the stage using an RC. The proposed RC increases the scanning speed by improving the control bandwidth of the system while maintaining the stability and the image quality.

2) In addition to improving the tracking performance, we show that the RC also reduces the interaction forces between the probe tip and the sample surface. Lower tapping forces help preserve the sample and lead to the longer use of the scanning probe.
3) We propose a new iterative approach for setting the tunable parameters of an RC in experimental settings. Using this approach, one can successfully determine the optimal parameters of an RC, resulting in better performance in AFM scanning than that of a conventional PI controller.

The following section provides the necessary background on the discrete-time implementation of an RC for the tapping mode AFM. The components of our AFM setup are introduced in Section III. In Section IV, we identify the dynamical characteristics of our stage controlled by the RC. The design and the analysis of the RC filters for the stage are given in Section V. The scanning experiments and the results are reported in Section VI. Finally, we conclude this study in Section VII with a discussion of the performed work.

\section{REPETITIVE CONTROL}

In Fig. 2(a), the RC-assisted PI control scheme for a tapping mode AFM is presented in the discrete time domain. The probe that appears in the diagram acts like a sensor, measuring the separation distance $\delta$ between the probe tip and the sample surface. Its dynamic response is approximately linear and much faster than that of the stage. Hence, it is considered to be a static device with a gain value equal to unity (i.e., $P(z)=1$ ) in our design. The nonlinearities in the probe dynamics and the ones due to the interactions between the probe tip and the sample surface are treated as unstructured uncertainties in our approach, which are handled by the robust design of RC. The surface topography and the initial distance between the tip and the sample are treated as disturbances $d$ to be rejected by the controller. The $q$ filter in the memory loop, which is a low-pass filter with a dc gain equal to 1 , is required to filter out the infinite loop gains that are introduced by the memory loop at the highfrequency harmonics. Basically, it defines the control bandwidth of the RC and also prevents the excitation of the undesired dynamics at high frequencies. The $b$ filter helps to maintain the inequality condition given in (1), which is sufficient for the stability of the RC. The function $R(\omega)$ is called the regeneration spectrum [23]

$R(\omega)=\left|q(z)\left[1-b(z) \frac{\operatorname{PI}(z) G(z)}{1+\operatorname{PI}(z) G(z)}\right]\right|<1$

$$
\text { where } z=e^{j \omega T} \text {. }
$$

Here, the frequency of interest varies from $0<\omega<\omega_{\max }$, where $\omega_{\max }=\pi / T$. The sampling time $T$ is typically selected as five to ten times smaller than the period of the highest frequency of interest. The stability condition given in (1) encourages the selection of the $b$ filter as simply the inverse of $\operatorname{PI}(z) G(z) /[1+\operatorname{PI}(z) G(z)]$ in order to make $R(\omega)$ as small as possible.

In order to improve the performance of the RC, a small sample advance $z^{n q}$ is incorporated into the $q$ filter to cancel out its negative phase. Similarly, a small sample advance $z^{n b}$ is incorporated into the $b$ filter to cancel out the negative phase of $\mathrm{PI}(z) G(z) /[1+\mathrm{PI}(z) G(z)]$ and consequently help to maintain 
(a)

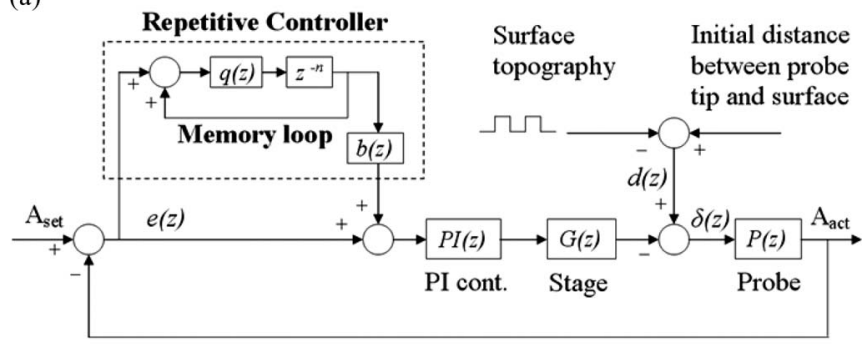

(b)

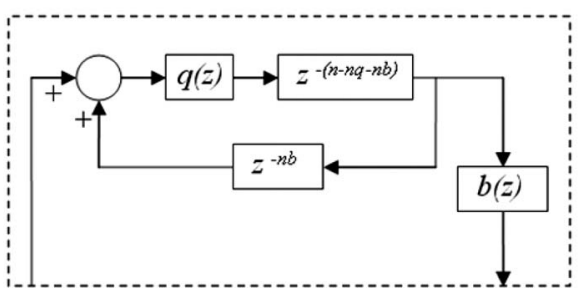

Fig. 2. (a) RC scheme for a tapping mode AFM. (b) Modified RC structure for incorporating the sample advances.

$R(\omega)<1$. Hence, the filters with sample advances can be written as

$$
q^{\prime}(z)=z^{n q} q(z) \quad \text { and } \quad b^{\prime}(z)=z^{n b} b(z) .
$$

These small sample advances can easily be absorbed in a much larger period of the exogenous input signal and do not cause a problem in implementation if the memory loop is modified as shown in Fig. 2(b). Although the values of $n b$ and $n q$ can be estimated from the phase diagrams of the filters directly, further adjustment is typically necessary to compensate for the additional phase lag in the closed-loop system due to the neglected dynamics of the probe. In this paper, we present a practical method for tuning of these parameters in AFM applications.

The stability and performance of the RC system are analyzed by the classical robust control approach [24], using the sensitivity $S$ and complementary sensitivity $T$ functions, which are defined in (3), where $L$ in (4) denotes the loop gain

$$
\begin{aligned}
& S=\frac{1}{1+L}, \quad T=\frac{L}{1+L} \\
& L=G\left(1+\frac{q}{1-q z^{-(n-n q)}} b z^{-(n-n q-n b)}\right) \mathrm{PI} .
\end{aligned}
$$

The sensitivity function $S$ is required to be small for good tracking (i.e., disturbance rejection) at operating frequencies that are far below the resonance frequency of the stage, $\omega_{\text {op }}<\omega_{\text {res }} / 10$, where the complementary function $T$ is required to be small for the frequencies around and above the resonance to achieve robust stability against unstructured modeling uncertainties (i.e., the neglected high-frequency dynamics) and also to attenuate the sensor noise.

\section{SETUP}

The sample to be scanned is placed on the piezoactuated $x-y-z$ nanostage (PI Inc., Germany, Model No. P-517.3CD),

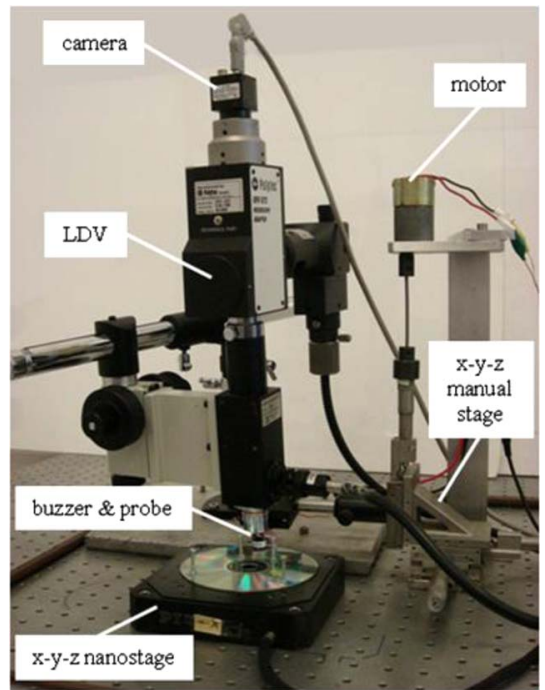

Fig. 3. Experimental setup for our tapping mode AFM.

which has a travel range of $100 \mu \mathrm{m} \times 100 \mu \mathrm{m} \times 20 \mu \mathrm{m}$ and is equipped with integrated capacitive sensors for precise positioning (resolution: $0.1 \mathrm{~nm}$ ). The stage is connected to a digital data acquisition (DAQ) card (PCI-DIO-96, National Instruments, Inc.) via a parallel $\mathrm{I} / \mathrm{O}$ port running a servo loop at $2 \mathrm{~ms} /$ cycle.

The scanning probe used in the experiments is suitable for tapping mode AFM operation, and its resonance frequency is around $350 \mathrm{kHz}$ (Olympus, OMCL-AC160TS). It is excited by a simple piezobuzzer. The vibration of the probe at the tip is measured by a laser doppler vibrometer (LDV) (Polytec $\mathrm{GmbH}$, Germany). The output of the LDV is first sent to an rms converter chip (Analog Devices, AD637JDZ) and then transferred to the computer through a separate DAQ unit (National Instruments USB 6251). The control signals for the raster scan motion on the $x-y$ plane and for imaging the surface along the $z$-axis are transferred to the nanostage through an amplifier. More details on the experimental setup (see Fig. 3) can be found in our earlier publication [25].

\section{IDENTIFICATION OF THE STAGE DyNAMICS}

We used step and impulse signals as input to characterize the dynamic response of our nanostage along the $z$-axis. A transfer function of the stage is constructed by the least-squares system identification technique. The best fit is obtained for a transfer function having a polynomial of third degree in the numerator and a polynomial of seventh degree in the denominator, as formulated as follows:

$G(z)=\frac{b_{3} z^{3}+b_{2} z^{2}+b_{1} z+b_{0}}{z^{7}+a_{6} z^{6}+a_{5} z^{5}+a_{4} z^{4}+a_{3} z^{3}+a_{2} z^{2}+a_{1} z+a_{0}}$. 


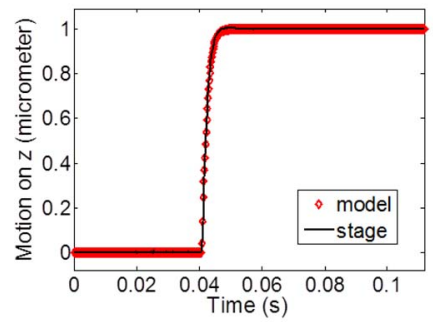

(a)

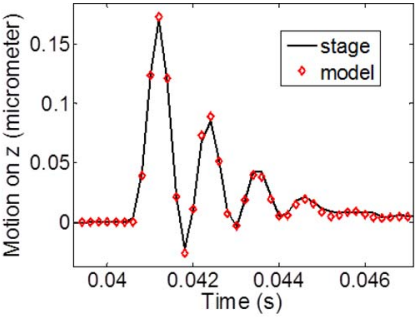

(b)
Fig. 4. (a) Step and (b) impulse responses of the stage and the model.

The coefficients of the polynomials are calculated using the method of least squares as for $k=1 \ldots N$

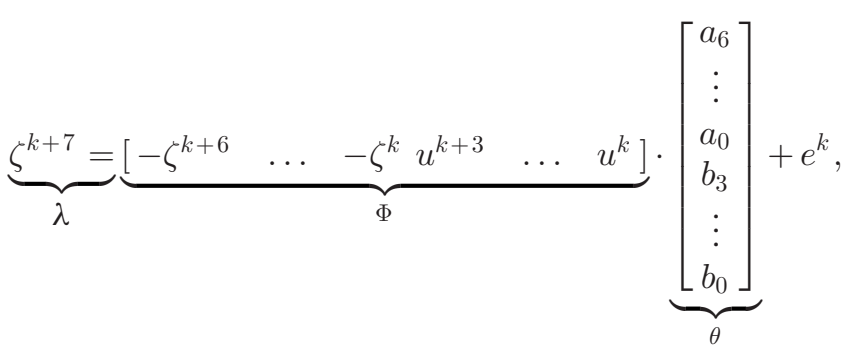

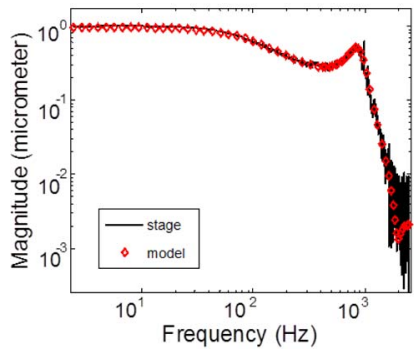

(a)

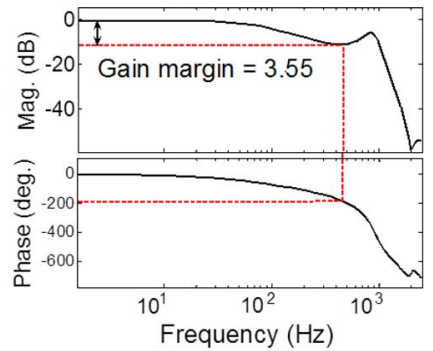

(b)
Fig. 5. (a) Frequency response curves and (b) bode plots of the stage and the model.

\section{Design And Analysis of the RC Filters}

\section{A. Design of the " $b$ " Filter}

We first tuned the PI controller shown in Fig. 2(a) for the best possible performance before adding the RC controller in series. Then, in order to make $R(\omega)<1$, the $b$ filter is chosen to be the inverse of $\operatorname{PI}(z) G(z) /[1+\operatorname{PI}(z) G(z)]$. As observed from (8), $G(z)$ has no nonminimum zeros; hence, it can be inverted safely. Furthermore, $\operatorname{PI}(z) G(z) /[1+\operatorname{PI}(z) G(z)]$ is multiplied by a low-pass filter having a dc gain equal to 1 to satisfy the causality requirement. The poles of this low-pass filter are chosen to be distinct and close to the center of the unit circle. The resulting transfer function for $b$ filter is given in (9), shown at the bottom of the page.

The effect of the $b$ filter on the dynamics of the system is shown in Fig. 6. Observe that the phase cannot be compensated well at frequencies higher than $100 \mathrm{~Hz}$ (see the red colored solid curve). While a noncausal optimal inverse can be calculated for the $b$ filter to further improve the phase compensation as suggested in [26], this problem is handled by adding a small sample advance $n b$ to the memory loop as already mentioned in Section II and will be further discussed in Section VI. The value of $n b=4$ is estimated from the phase diagram given in Fig. 6(a).

\section{B. Design of the " $q$ " Filter}

As mentioned earlier, the primary role of the $q$ filter is to reduce the negative effects of the noise in the system and the infinite gains introduced by the RC at the undesired harmonics of the input signal. For this reason, the cutoff frequency of the $q$ filter is chosen based on the mechanical bandwidth of the nanostage $(\sim 80 \mathrm{~Hz})$. Choosing a smaller value for the cutoff frequency would slow down the response of the RC while a higher value would cause the $\mathrm{RC}$ to replicate the undesired highfrequency signals. Obviously, an ideal low-pass filter should

$$
\begin{aligned}
G(z) & =\frac{0.0395 z^{3}+0.0707 z^{2}+0.0525 z+0.0121}{z^{7}-1.3400 z^{6}+1.1440 z^{5}-0.4713 z^{4}+0.0302 z^{3}-0.1892 z^{2}+0.1128 z-0.1100} \\
b(z) & =\frac{z^{8}-2.34 z^{7}+2.484 z^{6}-1.615 z^{5}+0.554 z^{4}-0.18 z^{3}+0.294 z^{2}-0.261 z+0.098}{0.047 z^{8}+0.045 z^{7}-0.0077 z^{6}-0.038 z^{5}-0.012 z^{4}+4.2 \times 10^{-8} z^{3}-5.1 \times 10^{-19} z^{2}}
\end{aligned}
$$




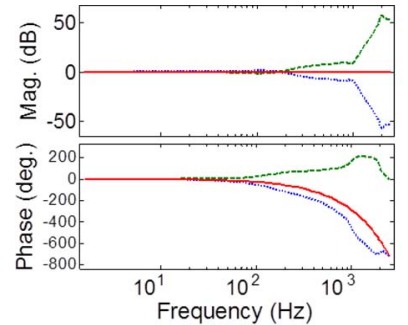

(a)

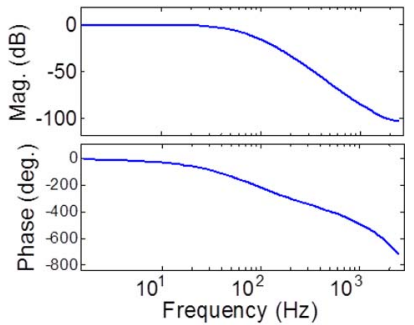

(b)
Fig. 6. (a) Bode plots of $b(z)$ (green dashed), $\operatorname{PI}(z) G(z) /[1+\operatorname{PI}(z) G(z)]$ (blue dotted), and $b(z) \operatorname{PI}(z) G(z) /[1+\operatorname{PI}(z) G(z)]$ (red solid); (b) bode plot of $q(z)$.

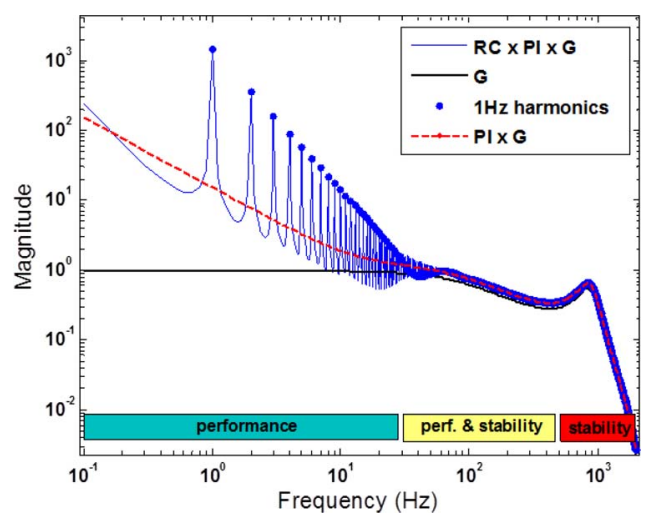

Fig. 7. Magnitude plots of the open-loop system including the stage, the stand-alone PI controller, and the RC.

have the gain value equal to 1 under the cutoff frequency, and zero above it. In order to converge to an ideal filter, we utilize a constant coefficient in the numerator of $q(z)$ and a polynomial with a high degree in the denominator [see (10)]. Note that choosing a polynomial for the denominator higher than fourth degree does not significantly improve the performance of our controller. The phase introduced by $q(z)$ is again compensated by adding a small sample advance $n q$ to the memory loop (see Section VI). The value of $n q=7$ is estimated from the phase diagram given in Fig. 6(b)

$$
q(z)=\frac{8.853 \times 10^{-5}}{z^{4}-3.612 z^{3}+4.892 z^{2}-2.945 z+0.6649} .
$$

\section{Analysis}

It is desirable that a controller introduces high loop gains into the closed-loop system without causing instabilities. According to the robust design, the magnitude of the sensitivity function for the closed-loop system must be less than 1 at low frequencies, where the tracking performance is required, and that of the complementary sensitivity function must be less than 1 at high frequencies, where robust stability is required. Note that it is not possible to satisfy both requirements at the same time neither at low nor at high frequency. However, there is a transition region in between, where both requirements can be satisfied up to a certain degree.

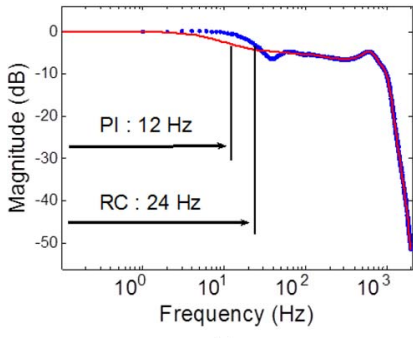

(a)

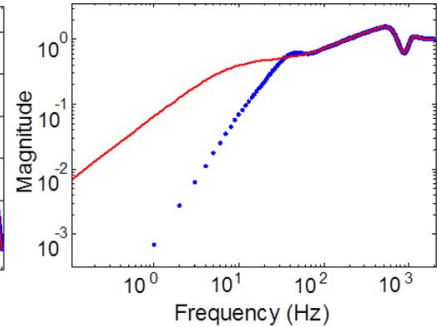

(b)
Fig. 8. (a) Magnitude plots of the closed-loop system under the stand-alone PI controller (red solid) and the RC (blue dotted). (b) Sensitivity functions of the closed-loop system under the stand-alone PI controller and the RC.

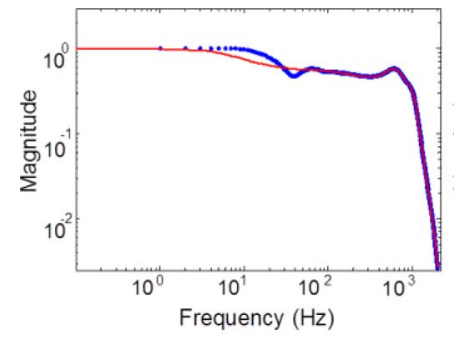

(a)

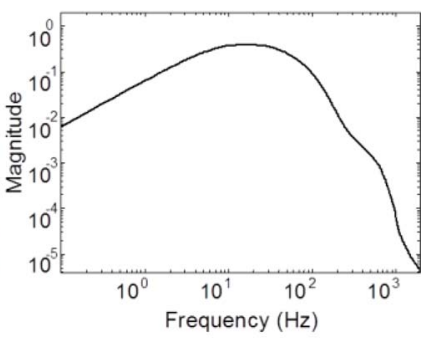

(b)
Fig. 9. (a) Complementary sensitivity functions of the closed-loop system under the stand-alone PI controller (red solid) and the RC (blue dotted.) (b) Reg neration spectrum of the closed-loop systems under the RC.

For the analysis of tracking performance and robustness of the RC utilizing the filters designed in earlier sections, the loop gain, sensitivity, and complementary sensitivity functions of the complete system [see Fig. 2(a)] are plotted and compared with those of the stand-alone PI controller in Figs. 7-9, respectively. The peaks in Fig. 7 are due to the high loop gains introduced by the $\mathrm{RC}$ at the fundamental frequency of $1 \mathrm{~Hz}$ and its harmonics (see blue dots). Fig. 8(a) shows that the closed-loop bandwidth (i.e., control bandwidth) of the $\mathrm{RC}$ is two times larger than that of the stand-alone PI controller for the same frequencies. One can observe that the sensitivity of the RC is much lower than that of the stand-alone PI controller [see Fig. 8(b)], suggesting a better tracking performance. The complementary sensitivities of both controllers (the stand-alone PI and the RC) decrease rapidly after the resonance frequency to maintain their robustness against the unstructured modeling uncertainties [see Fig. 9(a)]. Fig. 9(b) shows that the regeneration spectrum $R(\omega)$ is always less than 1 and the system is stable for all frequencies within the range of interest.

\section{EXPERIMENTS AND RESULTS}

\section{A. Tracking Performance of the Stage}

We first investigate the tracking performance of our stage along the $z$-axis by applying a square wave from a signal generator as the reference input. Hence, in this exercise, we do not consider the raster scan motion, the probe dynamics, and the force interactions between the probe and the sample at all. Our goal is to show that the RC improves the tracking performance of the stage significantly by reducing the rise time and 
(a)

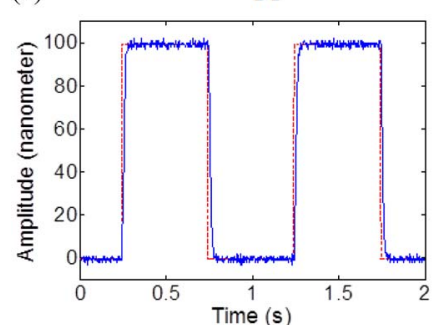

(c)

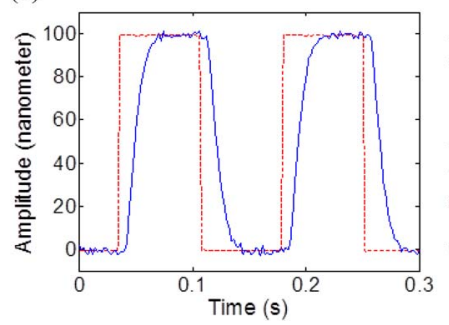

(b)

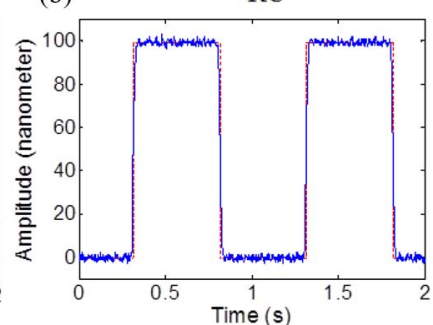

(d)

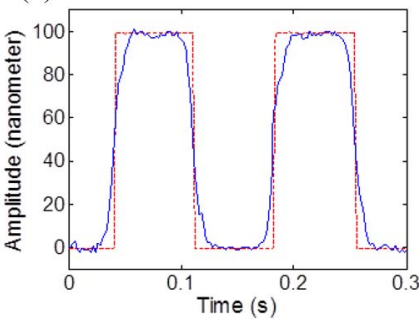

Fig. 10. Tracking performance of the stage under the stand-alone PI controller and the $\mathrm{RC}$ for the wave frequencies of $1 \mathrm{~Hz}$ [(a) and (b), respectively] and $7 \mathrm{~Hz}$ [(c) and (d), respectively].

compensating for the phase lag, which is not possible by using a conventional PI controller at high scanning speeds.

The amplitude of the square wave used in the tracking experiments is $100 \mathrm{~nm}$. The tracking experiments are performed at the wave frequencies of 1 and $7 \mathrm{~Hz}$. The proportional and integral gains of the stand-alone PI controller were set by trial and error for the wave frequency of $1 \mathrm{~Hz}$. The sample advances for the RC are taken as $n b=4$ and $n q=7$ (see Section V). The results of the experiments show that the tracking performance of the stage under the RC is $49 \%$ and $66 \%$ better than that of the stand-alone PI controller for the wave frequencies of 1 and $7 \mathrm{~Hz}$, respectively (see Fig. 10). The tracking performance is quantified based on the difference between the reference input (i.e., square wave) and the stage output (i.e., the actual trajectory followed by the stage).

The proposed RC assumes that the successive scan lines are similar and generate a control signal in the current scanned line based on the error made in the previous scan line. To investigate the performance of the RC when the successive scan lines are not similar, two simple experiments are designed. In both experiments, a square wave of varying amplitude (frequency = $1 \mathrm{~Hz}$ ) is tracked by the stage. For the sake of discussion, we assume that each step in the wave represents one scan line. Hence, the RC memorizes the control signal generated in the previous step while scanning the current one. In the first experiment, the amplitude of the square wave is suddenly increased from 5 to $80 \mathrm{~nm}$ [see Fig. 11(a) and (b)]. As shown in Fig. 11(b), the $\mathrm{RC}$ has no difficulty in tracking the desired trajectory since the magnitude of the control signal kept in the memory loop of the $\mathrm{RC}$ to track the short steps is relatively small and dissolves in the larger control signal generated by the PI controller in series to the RC when a higher step is encountered. In the second experiment, the amplitude of the square wave being tracked is suddenly reduced from 80 to $5 \mathrm{~nm}$. This time, we have observed some undesired peaks in the trajectory generated by the RC since

(a)
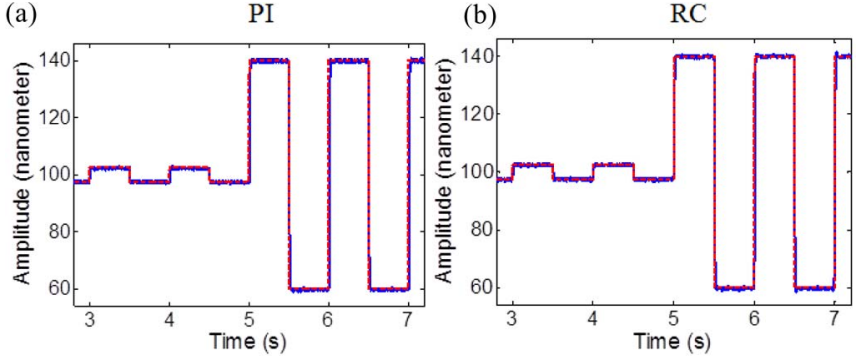

(c)

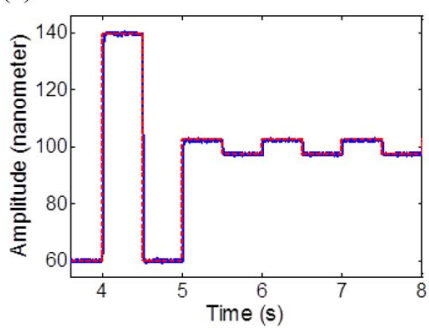

(d)

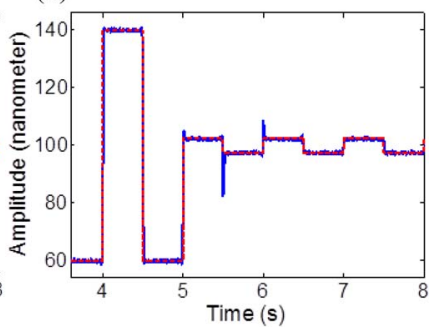

Fig. 11. Tracking performance of the stage under the stand-alone PI controller and the $\mathrm{RC}$ for the wave frequencies of $1 \mathrm{~Hz}$ when a large fluctuation occurs in the amplitude (for the sake of discussion, we assume that each step represents one scan line).

the control signal carried on by the RC from the previous step is now much larger than the small value desired for the current step [see Fig. 11(c) and (d)]. However, as shown in Fig. 11(d), the undesired peaks quickly disappear after the second short step. Moreover, such a large difference (i.e., 94\%) between the profiles of two consecutive scan lines is unexpected in real AFM scans if sufficiently small advances are made along the $y$-axis. We also note that such undesired peaks do not appear at all when there is less than $50 \%$ reduction in the amplitude.

\section{B. AFM Scanning}

The actual scanning experiments are conducted with a calibration grating having multiple steps (MikroMasch, TGZ02). The pitch and height of steps are $3.0 \mu \mathrm{m}$ and $82.5( \pm 1.5) \mathrm{nm}$, respectively. The calibration grating is placed on the $x-y-z$ nanostage. The tapping probe used in the experiments is initially brought close to the sample surface by means of a step motor (see Fig. 3). A distance of a few hundred nanometers typically remains between the probe tip and the calibration grating after the initial adjustment. This initial distance is then compensated automatically by the $z$-axis controller of the stage at the very beginning of the scan process. The probe is excited by a simple piezobuzzer to vibrate with an amplitude of $A_{\text {free }}=52 \mathrm{~nm}$ in the free air, where the set amplitude is chosen as $A_{\text {set }}=0.7 A_{\text {free }}$.

In our initial testing of the $\mathrm{RC}$ for scanning the grating, sudden peaks in the error signal $\left(A_{\text {set }}-A_{\text {act }}\right)$ are observed. They indicate an additional phase lag in the overall response of the system due to the neglected dynamics of the probe and the nonlinear force interactions between the probe tip and the scanned surface. To compensate for this additional lag, the small sample advances ( $n b$ and $n q$ ), initially estimated from the phase diagrams in Section II, are slightly altered to achieve the desired performance. We developed an iterative approach to estimate the 


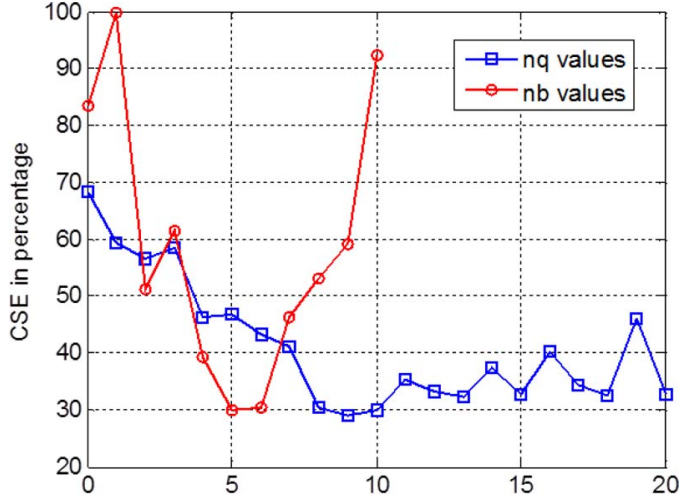

Fig. 12. Variation of $n b$ and $n q$ as a function of CSE (normalized).

(a)

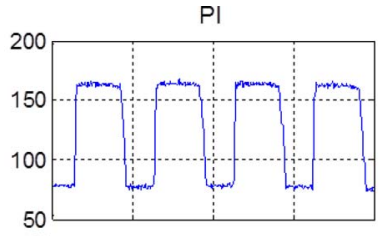

(b)

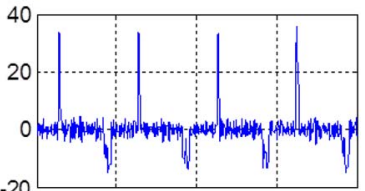

(c)
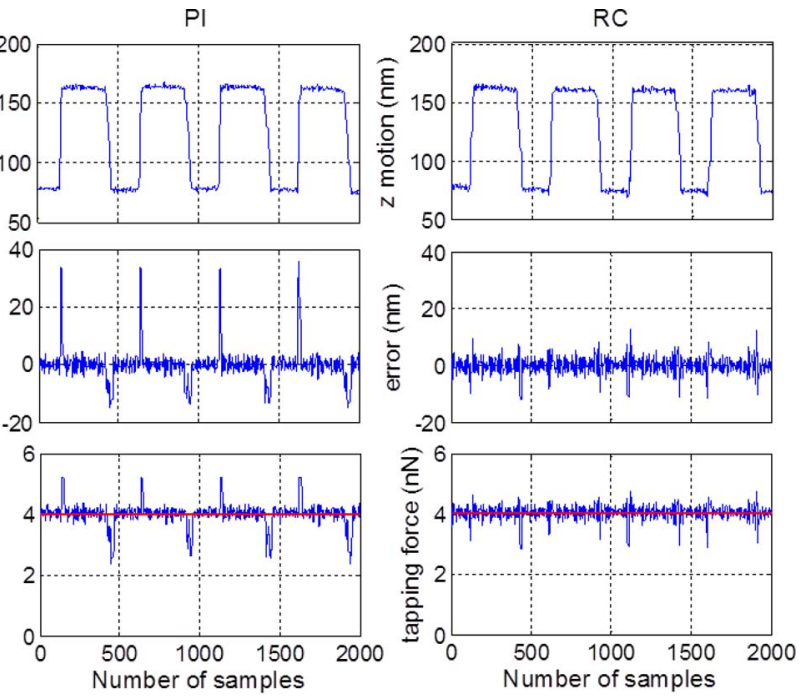

Fig. 13. Scan results for the stand-alone PI controller (left column) and the RC (right column) at the scanning speed of $3 \mu \mathrm{m} / \mathrm{s}$ : (a) scan profiles; (b) error signal; and (c) tapping forces.

optimal values of $n b$ and $n q$ resulting in the best performance. For this purpose, a new error measure, named as cumulative scan error (CSE), is defined to quantify the scan performance of the RC for the different combinations of $n b$ and $n q$ within a range. The CSE is calculated by using the error signal recorded during the scan $e(t)$, the scanning speed $v$, and the sampling time $T_{s}$, and then normalized by the step dimensions as

$$
\mathrm{CSE}=\frac{\sum|e(t)| T_{s} v}{h w} .
$$

The range of $n b$ and $n q$ values tested for the best performance is varied from 1 to 10 and 1 to 20, respectively, based on their initial estimates $(n b=4$ and $n q=7)$. In our iterative approach, first $n b$ is set to the initial value of 4 and the scanning experiments are performed for each integer value of $n q$. Then, the value of $n q$ returning the minimum scan error is chosen to search for the optimal value of $n b$ using the same approach. After several iterations, the optimal values of $n b$ and $n q$ are estimated as 5 and 9, respectively (see Fig. 12). As shown in Fig. 12, the scan error is more sensitive to variations in $n b$ than $n q$. (a)

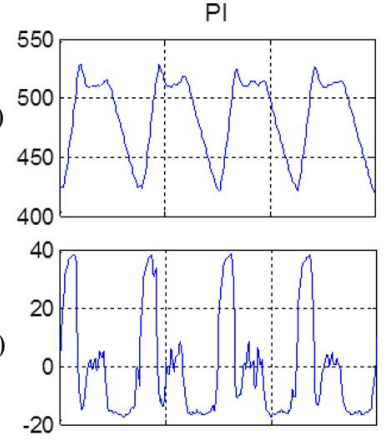

(c)
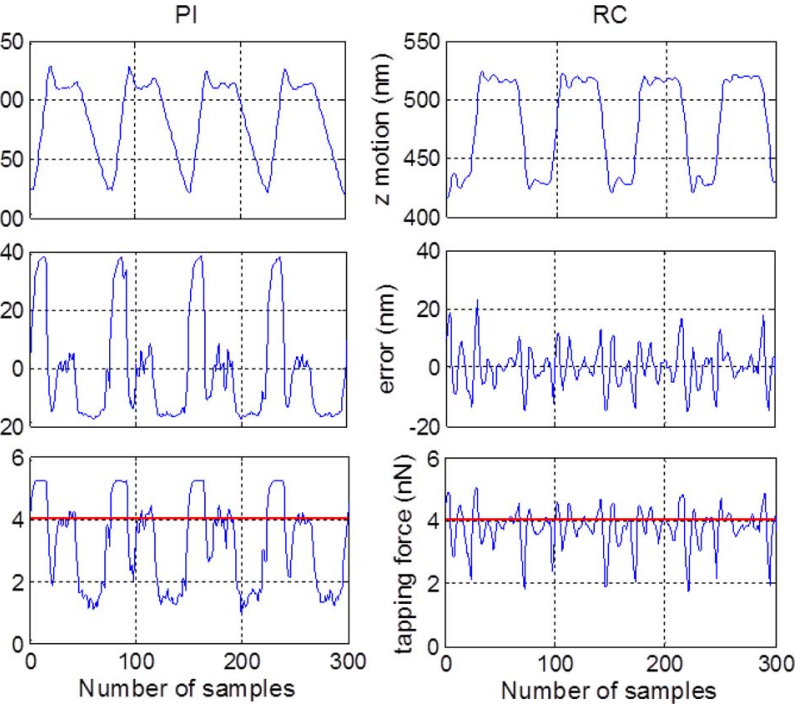

Fig. 14. Scan results for the stand-alone PI controller (left column) and the $\mathrm{RC}$ (right column) at the scanning speed of $21 \mu \mathrm{m} / \mathrm{s}$ : (a) scan profiles; (b) error signal; and (c) tapping forces.

Following the setting of $n b$ and $n q$, more comprehensive scanning experiments at different scanning speeds are performed and the performance of the $\mathrm{RC}$ is compared to that of the stand-alone PI controller using the CSE. The results presented in this paper are for the lowest and the highest scanning speeds of 3 and $21 \mu \mathrm{m} / \mathrm{s}$ (see Figs. 13 and 14, respectively). Since the pitch of the calibration steps used in the scanning experiments is $3 \mu \mathrm{m}$, these scanning speeds correspond to the wave frequencies of 1 and $7 \mathrm{~Hz}$ used in the experiments performed with the stage in the previous section. The results show that the CSE calculated for the RC is $30 \%$ less than that of the stand-alone PI controller at the scanning speed of $3 \mu \mathrm{m} / \mathrm{s}$. Although the scan profiles obtained by the RC and the stand-alone PI controller appear to match the desired profile very well, the phase delay in PI controller [see the peaks appearing in the error signal in Fig. 13(b)] results in larger CSE than that of the RC. This time delay further increases and causes even larger CSE for the stand-alone PI controller as the scanning speed is increased to $21 \mu \mathrm{m} / \mathrm{s}$. Compared to the stand-alone PI controller, the relative improvement achieved by the RC in scan profile was $66 \%$ at the scanning speed of $21 \mu \mathrm{m} / \mathrm{s}$ (see Fig. 14).

In addition to the tracking performance, the tapping forces observed in the RC are compared to that of the stand-alone PI controller. It is reported in [27] that the average tapping forces can be estimated as $\langle F\rangle=\left(A_{\text {free }}^{2}-A_{\text {set }}^{2}\right)^{1 / 2} k / Q_{\text {eff }}$, where $k$ is the stiffness of the cantilever probe and $Q_{\text {eff }}$ is its effective quality factor. To estimate the instantaneous tapping forces, the set amplitude is replaced by the instantaneous one in the previous formula. In our setup, $k=42 \mathrm{~N} / \mathrm{m}$ and $Q_{\text {eff }}=436$ for the probe and the set amplitude is $70 \%$ of the free air amplitude (i.e., $52 \mathrm{~nm}$ ), which results in a nominal tapping force of $4 \mathrm{nN}$ [see the red-colored horizontal lines in Figs. 13(c) and 14(c)]. Ideally, the controller should maintain the interaction forces between the probe tip and the sample surface at this nominal value for perfect imaging. As the tapping forces deviate from 
(a)

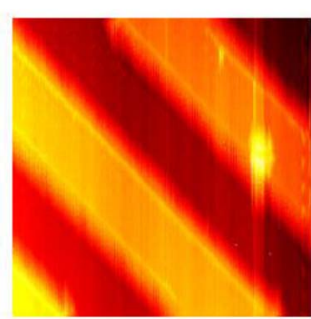

(c)

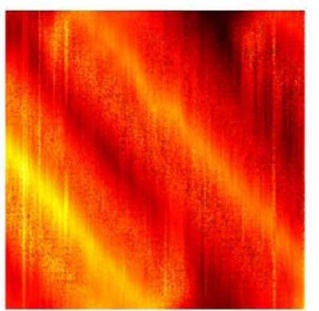

(b)

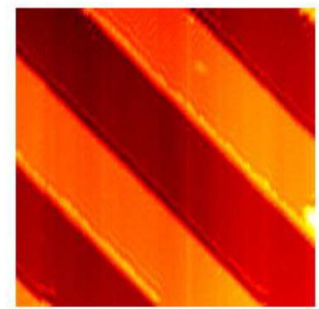

(d)

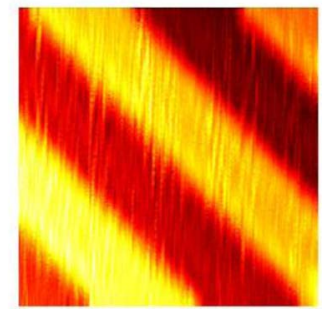

Fig. 15. The scan results for the stand-alone PI controller (left column) and the RC (right column) at the scan speeds of $3 \mu \mathrm{m} / \mathrm{s}$ (a and b) and $21 \mu \mathrm{m} / \mathrm{s}$ (c and d) when the calibration grating is rotated 45 degrees. The scanned area is $6 \mu \mathrm{m} \times 6 \mu \mathrm{m}$.

the nominal value, the image quality is reduced. The results show that the deviation of the tapping forces from the nominal value is small when the $\mathrm{RC}$ is used for scanning (compared to the stand-alone PI controller, a reduction of $40 \%$ and $60 \%$ is achieved for the scanning speeds of 3 and $21 \mu \mathrm{m} / \mathrm{s}$, respectively). Moreover, the average tapping forces above the nominal value are reduced by $5.5 \%$ and $58 \%$ for the scanning speeds of 3 and $21 \mu \mathrm{m} / \mathrm{s}$, respectively. In order to reduce the tracking errors and the tapping forces, the RC takes an early control action in the current scan line based on the control signal generated for the previous scan line.

To investigate the performance of the $\mathrm{RC}$ when the phase of the repetitive signal changes, we rotated the calibration grating by $45^{\circ}$ about the $z$-axis and scanned the resulting diagonal lines. Obviously, a slight phase difference occurs between the consecutive scan lines in this configuration as the stage advances along the $y$-axis. As shown in Fig. 15, the tracking errors due to this phase difference are compensated well by the RC controller, but not by the stand-alone PI controller (see Fig. 15).

\section{DISCUSSION AND CONCLUSION}

We implemented an RC to achieve better performance than the conventional PI controller in scanning nanoscale surfaces at high scanning speeds without sacrificing the image quality. Moreover, we showed that the magnitude of the tapping forces between the scanning probe and the surface was significantly decreased by the RC. For example, when the scanning speed was increased by sevenfold, the scan error and the average tapping forces were reduced by $66 \%$ and $58 \%$, respectively. The reduction in the magnitude of tapping forces is important since high values can easily damage the specimen (especially, the biological ones) as well as the scanning probe.

The scan performance achieved by the $\mathrm{RC}$ is better than the stand-alone PI controller since the successive scan lines are similar and the proposed RC takes into account the knowledge of the previous scan line while scanning the current one. The similarity of the successive scan lines is certainly true for a sample having a smooth surface profile but also holds for a rougher surface since the topography being measured is smoothened by the probe tip due to its finite radius and cone angle [12]. In the stand-alone PI controller, when the probe encounters the rising edge of a step, the vibration amplitude of the probe reduces to zero rapidly and sticking occurs, which appears as a sudden peak in the error signal [see Figs. 13(b) and 14(b)]. The sticking is almost unavoidable since the control signal calculated in the current servo loop based on the error signal is only effective in the next servo loop. On the other hand, the RC can take a control action in the current scan line based on the error estimated for the previous scan line. As a result, we have less sticking, smaller error, and smaller tapping forces compared to the stand-alone PI controller. When the probe encounters the falling edge of the same step, it suddenly detaches from the sample surface and the magnitude of the error cannot exceed $A_{\text {set }}-A_{\text {free }}$, which limits the speed of the response and, hence, the tracking performance of the stand-alone PI controller. It takes longer time for the probe oscillations to reach the desired amplitude $A_{\text {set }}$ again. Hence, the resulting scan profile during the saturation period is erroneous. On the other hand, the error signal entering to the PI controller shown in Fig 2(a) is augmented by the RC, and hence, the error saturation is eliminated and a faster response is achieved [see Fig. 13(a)].

While the performance of the $\mathrm{RC}$ is better than that of the stand-alone PI controller in many aspects, one must know the period of the repetition for its implementation. In our case, this period is the time spent for the back and forth motions of the stage along a single scan line. Since the user sets the period of this raster scan motion, the aforementioned requirement can be satisfied easily. Hence, the proposed RC only requires that the successive scan lines to be repetitive, but their profiles do not have to be periodic. We should also mention that the implementation of the RC requires small sample advances ( $n b$ and $n q$ ) in the memory loop to compensate for the phase delays introduced by the filters $b$ and $q$ and the closed-loop system itself. Their selection is critical for achieving better performance, especially at high scanning speeds. However, we showed that the iterative approach proposed in Section VI could successfully determine the optimal values of $n b$ and $n q$ once the good initial estimates were provided. Those initial values can easily be obtained from the phase diagrams of the associated filters.

\section{REFERENCES}

[1] G. K. Binnig, C. F. Quate, and C. Gerber, "Atomic force microscope," Phys. Rev. Lett., vol. 56, pp. 930-933, Mar. 1986.

[2] D. Y. Abramovitch, S. B. Andersson, L. Y. Pao, and G. Schitter, "A tutorial on the mechanisms, dynamics, and control of atomic force microscopes,' in Proc. Am. Control Conf. New York City, Jul. 2007, pp. 3488-3502.

[3] T. Ando, T. Uchihashi, and T. Fukuma, "High-speed atomic force microscopy for nano-visualization of dynamic biomolecular processes," Prog. Surface Sci., vol. 83, pp. 337-437, Nov. 2008. 
[4] H. Yamashita, K. Voïtchovsky, T. Uchihashi, S. A. Contera, J. F. Ryan, and T. Ando, "Dynamics of bacteriorhodopsin 2D crystal observed by highspeed atomic force microscopy," J. Struct. Biol., vol. 167, pp. 153-158, May 2009

[5] M. Shibata, H. Yamashita, T. Uchihashi, H. Kandori, and T. Ando, "Highspeed atomic force microscopy shows dynamic molecular processes in photoactivated bacteriorhodopsin," Nat. Nanotechnol., vol. 5, pp. 208212, Mar. 2010

[6] G. Schitter, K. J. Aström, B. E. DeMartini, P. J. Thurner, K. L. Turner, and P. K. Hansma, "Design and modeling of a high-speed AFM scanner," IEEE Trans. Control Syst. Technol., vol. 15, no. 5, pp. 906-915, Sep. 2007.

[7] G. Schitter, P. Menold, H. F. Knapp, F. Allgöwer, and A. Stemmer, "High performance feedback for fast scanning atomic force microscopes," Rev. Sci. Instrum., vol. 72, no. 8, pp. 3320-3327, Aug. 2001.

[8] S. Salapaka, A. Sebastian, J. P. Cleveland, and M. V. Salapaka, "High bandwidth nano-positioner: A robust control approach," Rev. Sci. Instrum., vol. 73, no. 9, pp. 3232-3241, Sep. 2002.

[9] Y. Li and J. Bechhoefer, "Feedforward control of a piezoelectric flexure stage for AFM," in Proc. Amer. Control Conf., Seattle, Jun. 2008, pp. 2703-2709.

[10] L. Y. Pao, J. A. Butterworth, and D. Y. Abramovitch, "Combined feedforward/feedback control of atomic force microscopes," in Proc. Amer. Control Conf., New York, Jul. 2007, pp. 3509-3515.

[11] T. Uchihashi, N. Kodera, H. Itoh, H. Yamashita, and T. Ando, "Feed forward compensation for high-speed atomic force microscopy imaging of biomolecules," Jpn. J. Appl. Phys., vol. 45, no. 3B, pp. 1904-1908, Mar. 2006.

[12] G. Schitter, R. W. Stark, and A. Stemmer, "Fast contact-mode atomic force microscopy on biological specimen by model-based control," Ultramicroscopy, vol. 100, pp. 253-257, 2004.

[13] H. Fujimoto and T. Oshima, "Nanoscale servo control of contact-mode AFM with surface topography learning observer," in Proc. 10th IEEE Int. Workshop Adv. Motion Control, Trento, Mar. 2008, pp. 568-573.

[14] Y. Wu, Q. Zou, and C. Su, "A current cycle feedback iterative learning control approach for AFM imaging," IEEE Trans. Nanotechnol., vol. 8, no. 4, pp. 515-526, Jul. 2009.

[15] U. Aridogan, Y. Shan, and K. K. Leang, "Design and analysis of discretetime repetitive control for scanning probe microscopes," ASME J. Dyn. Syst., Meas. Control, vol. 131, no. 061103, Nov. 2009.

[16] T. Inoue, S. Iwai, and M. Nakano, "High accuracy control of a proton synchrotron magnet power supply," in Proc. 8th IFAC World Congr., Kyoto, Aug. 1981, part 3, pp. 3137-3142

[17] B. A. Francis and W. M. Wonham, "The internal model principle for linear multivariable regulators," Appl. Math. Optim., vol. 2, pp. 170-194, May 1975.

[18] M. Steinbuch, "Repetitive control systems with uncertain period-time," Automatica, vol. 38, pp. 2103-2109, Jun. 2002.

[19] S. Hara, Y. Yamamoto, T. Omata, and M. Nakano, "Repetitive control systems: A new type servo system for periodic exogenous signals," IEEE Trans. Autom. Control, vol. 33, pp. 657-667, Jul. 1988.

[20] G. Weiss, "Repetitive control systems: Old and new ideas," in Systems and Control in the 21st Century, C. Byrnes, B. Datta, D. Gilliam, and C. Martin, Eds. Boston, MA: Birkhäuser, 1997, vol. 22, pp. 389404

[21] L. Guvenc, "Stability and performance robustness analysis of repetitive control systems using structured singular values," ASME J. Dynam. Syst., Meas. Control, vol. 118, pp. 593-597, Sep. 1996.

[22] Y. Wang, F. Gao, and F. J. Doyle, "Survey on iterative learning control, repetitive control, and run-to-run control," J. Process Control, vol. 19, pp. 1589-1600, Dec. 2009.

[23] K. Srinivasan and F. R. Shaw, "Analysis and design of repetitive control systems using the regeneration spectrum," ASME J. Dynam. Syst., Meas. Control, vol. 113, pp. 216-222, Jun. 1991.

[24] B. A. Guvenc and L. Guvenc, "Robust repetitive controller design in parameter space," ASME J. Dynam. Syst., Meas.Control, vol. 128, pp. 406413, Jun. 2006.

[25] I. Gunev, A. Varol, S. Karaman, and C. Basdogan, "Adaptive Q control for tapping mode nanoscanning using a piezoactuated bimorph probe," Rev. Sci. Instrum., vol. 78, p. 043778, Apr. 2007.

[26] Q. Zou and S. Devasia, "Preview-based optimal inversion for output tracking: Application to scanning tunneling microscopy," IEEE Trans. Control Syst. Technol., vol. 12, no. 3, pp. 375-386, May 2004.

[27] T. R. Rodriguez and R. Garcia, "Theory of Q control in atomic force microscopy," Appl. Phys. Lett., vol. 82, no. 26, pp. 4821-4823, Jun. 2003.

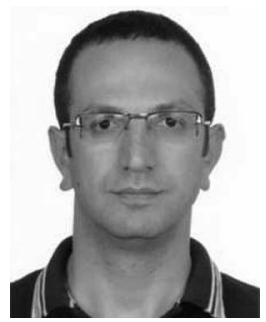

Serkan Necipoglu received the B.S. degree in mechanical engineering from Yildiz Technical University, Istanbul, Turkey, in 2002, and the M.S. degree in control systems from Imperial College London, London, U.K., in 2004. He is currently a graduate student in the Mechanical Engineering Program of Istanbul Technical University, Istanbul, Turkey.

His research interests include control systems, system dynamics, mechatronics, and nanotechnology.

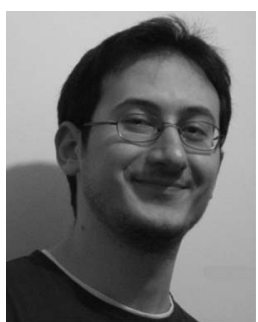

Selman A. Cebeci received the B.S. degree in mechanical engineering from Sabanci University, Istanbul, Turkey, in 2008 . He is currently a graduate student in the Department of Mechanical Engineering, Koc University, Istanbul.

His research interests include control systems, mechatronics, and nanotechnology.

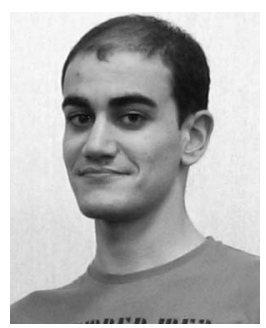

Yunus E. Has received the B.S. degree in mechanical engineering from Baskent University, Ankara, Turkey, in 2008. He is currently a graduate student in the Department of Mechanical Engineering, Koc University, Istanbul, Turkey.

His research interests include control systems, mechatronics, and nanotechnology.

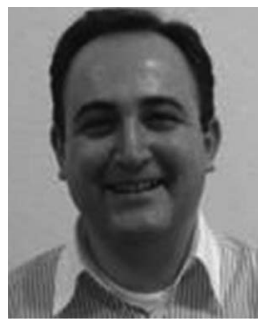

Levent Guvenc received the B.S. degree in mechanical engineering from Bogazici University, Istanbul, Turkey, in 1985, the M.S. degree in mechanical engineering from Clemson University, Clemson, SC, in 1988, and the Ph.D. degree in mechanical engineering from the Ohio State University, Columbus, in 1992.

$\mathrm{He}$ is currently a Faculty Member in the Department of Mechanical Engineering, Istanbul Technical University, Istanbul, Turkey, and the Director of the Automation Lab and the EU funded Automotive Controls and Mechatronics Research Center. His current research interests include automotive control and mechatronics, helicopter stability and control, mechatronics, and applied robust control.

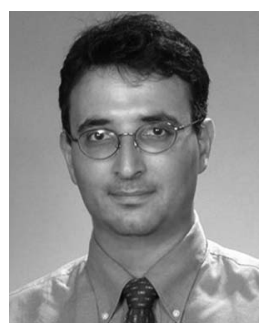

Cagatay Basdogan received the Ph.D. degree in mechanical engineering from Southern Methodist University, Dallas, TX, in 1994

$\mathrm{He}$ is currently a Faculty Member in the Mechanical Engineering and Computational Sciences and Engineering Programs of Koc University, Istanbul, Turkey, and. also the Director of the Robotics and Mechatronics Laboratory, Koc University. Before joining to Koc University, he was with NASA-JPL/Caltech, MIT, and Northwestern University Research Park. His research interests include haptic interfaces, robotics, mechatronics, biomechanics, medical simulation, computer graphics, and multimodal virtual environments.

He is currently an Associate Editor of the IEEE TRANSACTIONS ON HAPTICS AND COMPUTER ANIMATION and Virtual Worlds journals. 